\title{
A Web-based System for Shelter and Evacuation Path Selection Using Spatial Models in Disaster Situations
}

\author{
Hyun-Suk Hwang ${ }^{1}$, Il-Kyeun $\mathrm{Ra}^{2}$ and Chang-Soo Kim ${ }^{3 *}$ \\ 'Interdisciplinary Program of Information Systems, Pukyong National University, \\ Busan 608-737, Korea,hhs@pknu.ac.kr \\ ${ }^{2}$ Department of Computer Science and Engineering, University of Colorado \\ Denver, CO 80203, USA, ilkyeun.ra@ucdenver.edu \\ ${ }^{3}$ Department of IT Convergence and Application Engineering, Pukyong National \\ University, Busan 608-737, Korea, cskim@pknu.ac.kr
}

\begin{abstract}
Evacuation to disaster shelters can be optimized by giving instructions to evacuees to help them find the most suitable shelters and evacuation paths from their location. To generate such information in real-time, construction of spatial databases and complex spatial analysis models through Web Map Services (WMS) and Web Feature Services (WFS) based on Open Geospatial Consortium Web Services (OWS) is required. Focusing on the implementation of distributed OWS resources to provide more immediate information in the case of an emergency, we propose a Web-based system that identifies suitable shelters and provides the shortest evacuation path to each shelter for civilians who are within the danger zone. Specifically, (1) a search framework based on the OWS architecture is proposed and utilized to develop both Web-based and smartphone applications connected with WMS types of spatial data on the Web; (2) the spatial analysis models proposed include three sub-models, namely, data construction, shelter selection using scores by natural breaks, and an evacuation path model using network analysis. We identify candidate shelters by considering proximity to health care facilities and to major road, and coverage of each shelter in danger zone.
\end{abstract}

Keywords: Disaster Management Systems, GIS systems, Spatial Data Infrastructure, Spatial Models, OGC Web Services

\section{Introduction}

The main goal of evacuation routing planning is to guide all evacuees in a safe area to prevent lots of loss of life from natural disasters, so it is necessary to construct an emergency preparedness system to find shelters and its shortest routes. Busan Metropolitan City in South Korea, whose buildings and houses are encompassed by mountains or coasts, and areas are in densely populated regions with windy and intensive floods, has designated several sites for emergency shelters to prepare natural disasters such as flooding, wave storms, landslides, and windstorms. Several facilities here are appropriately regarded as temporary shelters including schools, churches, and other public facilities due to their traditional use as venues for community gatherings [1].

Evacuation to these shelters must be performed in a timely manner to minimize casualties in natural disasters or terrorist attacks. Shekhar [2] developed the Capacity Constrained Route Planner (CCRP) generalizing shortest path algorithms by considering the spread of people over space and time. Moreover, selecting shelters and generating routing paths requires a significant amount of processing and manipulation of several data sources. Therefore, servers are needed with spatial data processing capability, and only

* Corresponding Author : Chang-Soo Kim(cskim@pknu.ac.kr) 
their results should be sent to clients through GIS applications based on Web Services [34].

With the advance of technology in spatial data creation, spatial data have been created and owned by many different agencies that utilize them to satisfy their own needs [5-6]. The fragmentation of spatial data owners causes diversity in policies related to spatial data, standards, and the tools used to manage and coordinate these data. The diversity of approaches to data coordination leads to inconsistency and heterogeneity within spatial data from multiple-sources. Therefore, the diversity of spatial data requires a shared platform to facilitate access to and integration of data using a spatial data infrastructure [5, 7]. Distributed data can be approached within a framework that maintains standards, access to, and the interaction of spatial data, and also enables results to be presented on multiple platforms, including smartphones and Web applications, to allow users to easily discover appropriate shelters and routes.

We propose a system that includes the following technologies and spatial analysis models: (1) an OWS-based framework to enhance the search for spatial data extracted from multiple agencies; (2) spatial analysis models to decide shelters and path routes based on a user's location for emergency situations such as flooded and broken housing caused by intensive floods or landslides; and (3) an application development based on a physical architecture to provide evacuation paths through an easier interface by interoperating Application servers and Web servers with different types of spatial data.

\section{Methodology}

\subsection{Web-Based Gis Applications}

There are several research studies that have used Open Geospatial Consortium (OGC) standards for performing data interoperation between spatial data sources using Web services [4, 8-9]. For instance, the Andean Information System for Disaster Prevention and Relief [7][10] is an initiative promoted by the Andean Committee, which supports tools for discovery, visualization, and access to data related to disaster risks. The system is available to different organizations within the countries of the Community of Andean Nations, including Bolivia, Columbia, Ecuador, and Peru. The following main system requirements have been identified: (1) decentralization to offer autonomy and independence for data producers to use and manage their own data; (2) accessibility to offer access to the system using Web services with enabled devices; and (3) sustainability to implement a technical solution with acceptable costs. However, the system providing the search and viewing services does not include a guidance service to search shelters and routing paths for an evacuation process in the interoperated datasets.

Another example is the PREVIEW global data risk platform [11], which aims to facilitate the process of data sharing and dissemination using OGC standards. PREVIEW is useful in assessing the risk of a disaster's range on a global scale. The system has several capabilities, which include the following: (1) a portal service that enables data discovery; (2) a catalogue that enables searching for detailed information contained in the data; and (3) a process that enables data to be used in spatial analysis. This research confirms that spatial data are useful in data integration and processing through OGC standards.

\subsection{Shelter Selection and Evacuation Path Model}

It is important to determine evacuation shelters in emergency situations caused by damages of natural disasters such as floods, wave storms, landslides, and windstorms, because maintaining several sites solely for shelters is costly. Therefore, a cost effective solution is to identify existing facilities such as community centers, 
churches, and schools as shelters, which are physically and socially suitable for temporary evacuation shelters.

Several studies have been conducted to determine variables to support shelter selection for evacuation using GIS models. Lee [12] proposed a proximity method to highways and evacuation routes using road structure. Karr and Hodgson [1] researched a method to rank existing and candidate shelters such as schools, colleges, churches, and community centers based on their site suitability. Sanyal and $\mathrm{Lu}$ [13] researched the closest shelter covering the most affected area. Chen [14] proposed a model for optimizing the plan of earthquake shelters considering financial constraints and evacuation efficiency using three-level hierarchical location model.

Papinski and Scott [15] illustrate the process for routes captured using GPS tracking to compare observed routes with the shortest routes using network analysis. A network is a system of interconnected elements such as edges and connecting junctions, which represents possible routes from one location to another. Network analysis is a procedure that navigates through the connectivity of transportation networks to yield meaningful results such as finding all elements upstream of a point or the shortest path between two points.

The spatial analysis models are executed through a geoprocessing service on Web services $[9,16-18]$. Web clients are lightweight applications that are only able to send packets of simple data, such as text, numbers, and uncomplicated geographic features, to a Web server.

\section{Proposed Spatial Model Description}

\subsection{Data Sources}

We designed a database scheme with focus on the spatial analysis models as shown in Table 1, which provide shelters and an evacuation path to each shelter. First, the model must contain original data sources, such as buildings, damaged areas, and road network.

The STD_BUDG_AS object expresses the records of all the building in the district of related areas. The data will be filtered to extract suitable candidates, such as schools or churches, in the data construction model; hospital data are also included. The LY_DAMAGE_AS object includes the damaged area generated from previous disasters. The ROAD_NETWORK object includes the network datasets of road information, which are used in the route analysis process.

The datasets for the shelter and evacuation path selection model are labelled as LY_CANDIDATE_nHOSPITAL_PS, LY_CANDIDATE_nROAD_PS, and LY_FINALSHELTER_PS. The LY_FINALSHELTER_PS contains information about the location of the appropriate shelters. Each score is generated from their proximity to hospitals and roads based on the defined danger zone. The LY_CANDIDATE_nHOSPITAL_PS includes information regarding hospitals in proximity to the candidate shelters and contains fields such as NEAR_ID, NEAR_DIST, and SCORE. LY_CANDIDATE_nRoad_PS includes information regarding roads in proximity to the candidate shelters and contains fields such as NEAR_ID, NEAR_DIST, and SCORE. LY_THIESSEN_AS contains information about the INPUT_FID and AREA of Thiessen areas for each of the allocated shelters within the defined damage area. 
Table 1. Spatial Data Resources

\begin{tabular}{lll}
\hline Category & \multicolumn{1}{c}{ Tables } & \multicolumn{1}{c}{ Data Fields } \\
\hline \multirow{2}{*}{ Basic } & STD_BLDG_AS & Building name, road name \\
Spatial & STD_RDCT_LS & Road class \\
Data & LY_DAMAGE_AS & Building and road name, score \\
& LY_HOSPITAL_PS & Hospital name, road name, zip code \\
\hline Shelter & LY_CANDIDATE_nHOSPITAL_PS & Near feature ID and distance, score \\
Selection & LY_FINALSHELTER_PS & Score \\
Data & LY_THIESSEN_AS & Input feature ID, area of allocated shelters \\
\hline \multirow{2}{*}{ Network } & ROAD_NETWORK & Road code, road name \\
Data & LY_CANDIDATE_nROAD_PS & Near feature ID and distance, score \\
& LY_ROUTE_LS & Facility ID, incident and facility approach \\
\hline
\end{tabular}

\subsection{Spatial Analysis Model}

3.2.1. Data Construction Model: The data construction model in the Figure 1 identifies candidate shelters, which include existing schools, colleges, churches, and community centers. To find target candidates, we used two factors: (1) Proximity to road networks is considered by highlighting buildings located near a major transportation route with more than two lanes; (2) Proximity to health care facilities is considered to treat evacuees who require medical attention.

Executing this model depends on changes made to the building, hospital, and road data. The letter "P" on top of the COM.STD_BLDG_AS building data indicates a parameter users can modify when executing the model. The building data are copied using the "Feature class to feature class" custom tool. Each copied feature is added to a "SCORE" field using the "Add Field" default tool. The results of candidate shelters are processed using the "Near" tool to generate the nearest distance from each hospital and road within the range of the defined search radius.

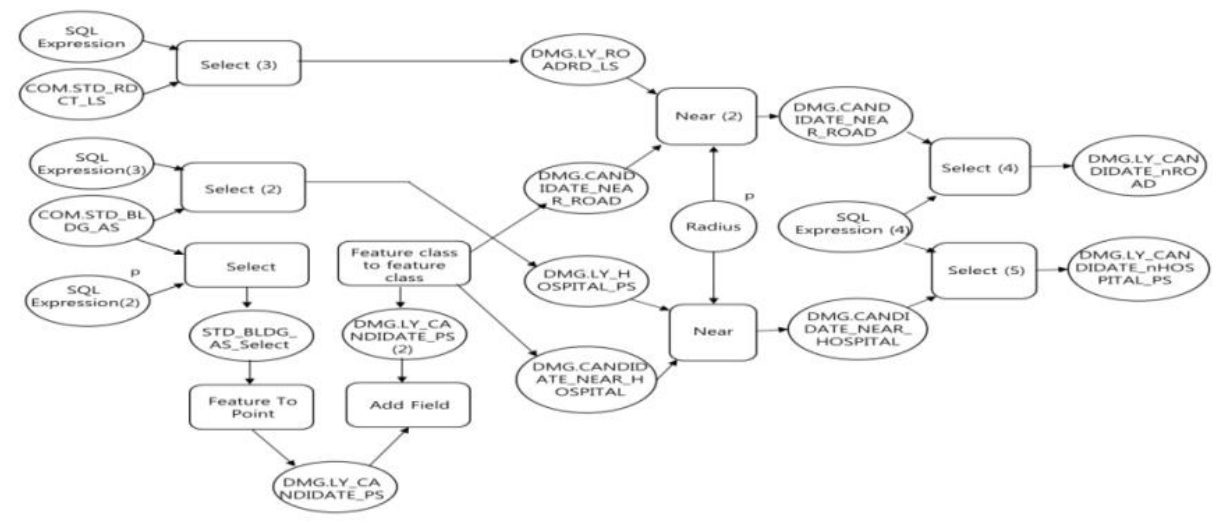

Figure 1. Diagram of the Data Construction Model

3.2.2. Shelter Selection Model: We suggest three main factors as shown in the Table 2 , which is proximity to health care facilities, proximity to roads, and coverage of each shelter in a danger zone. These factors are based on other studies [1, 12, 14, 19] such as shelter suitability models. 
Table 2. Factors in our Shelter Suitability Model

\begin{tabular}{lll}
\hline Factors & Comment & Related Data Sources \\
\hline Danger zone & Locations in dangerous zone are excluded & LY_DAMAGE_AS \\
\hline $\begin{array}{l}\text { Proximity to health care } \\
\text { facility }\end{array}$ & $\begin{array}{l}\text { Locations closer to health facility are more } \\
\text { suitable }\end{array}$ & $\begin{array}{l}\text { STD_BLDG_AS } \\
\text { LY_HOSPITAL_AS }\end{array}$ \\
\hline Proximity to major road & $\begin{array}{l}\text { Locations closer to major transportation } \\
\text { road are more suitable }\end{array}$ & LY_ROADRO_LS \\
\hline $\begin{array}{l}\text { Coverage of each shelter in a } \\
\text { danger zone }\end{array}$ & Wider range is more suitable & $\begin{array}{l}\text { LY_CANDIDATE_PS } \\
\text { LY_THIESSEN_AS }\end{array}$ \\
\hline
\end{tabular}

We use three factors: proximity to hospitals and roads and shelter coverage for defined dangerous regions. The rating of each factor is multiplied by its respective weight in each dangerous region, and the weighted values are combined by addition.

Users can obtain information regarding the final shelters depending on their parameters, which are damaged areas, radius of damaged area, and number of shelters, using this model. The model utilizes the letter "P" on the right side of related variables. This model in Figure 2 is categorized into three steps: clip steps to be bounded from the damaged area, erase steps to delete candidate shelters within the damaged area, and scoring steps to calculate the score of each shelter according to each factor rating. The score can be denoted for each factor as in (1)

$$
\begin{aligned}
\operatorname{Score}_{i}(k)= & \sum_{j=1}^{n}\left[F R_{j}(k) * w_{j}(i)\right] \\
& =F R_{1}(k) * w_{1}(i)+F R_{2}(k) * w_{2}(i)+---+F R_{n}(k) * w_{n}(i)
\end{aligned}
$$

where $\operatorname{Score}_{i}(k)$ is the score for each shelter $\mathrm{k}$ in a dangerous zone i. $F R_{j}(k)$ is the factor rating of each shelter for factor $\mathrm{j}, \mathrm{n}$ is the number of factors, and $w_{j}(i)$ is the weight assigned to factor $\mathrm{j}$ in a danger zone such that $\sum_{j=1}^{n} w_{j}=100$.

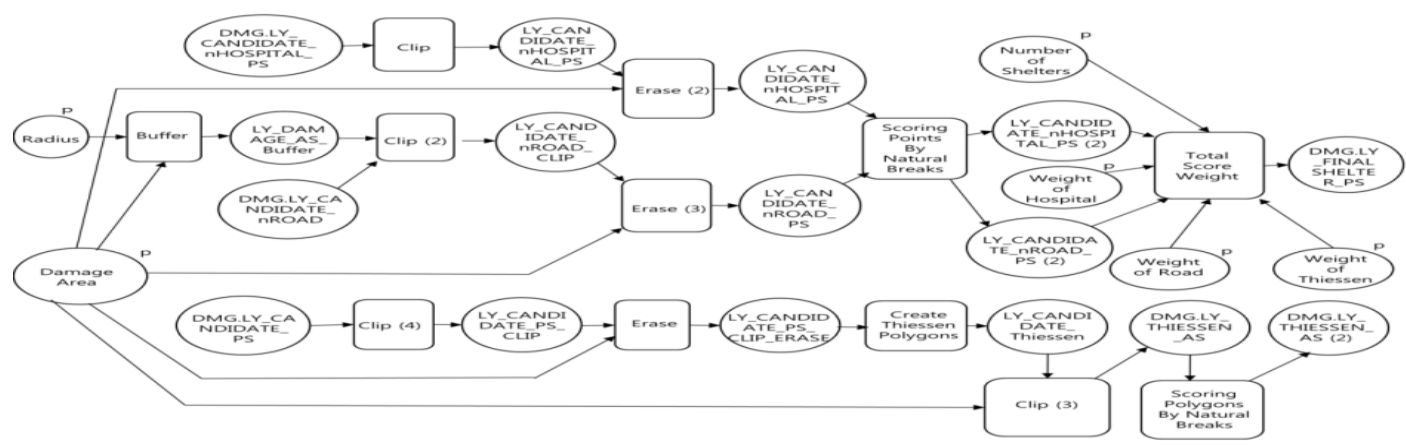

Figure 2. Diagram of the Shelter Selection Model

3.2.3. Evacuation Path Model: After the final shelters are selected, the next step is to create a route to each shelter based on the network analysis. Two input parameters are needed, namely, current location which describes a user's current location, and the number of final shelters, which are the output of the shelter selection model and one output file to store routes. This model uses the closest facility method which takes two variables, "Stops" and "Facilities", and calculates the shortest route from each stop to each facility.

There are several operation tools in this model as shown in Figure 3. The Make Closest Facility Layer is used for the process of making the layer of selected shelters. The Add Shelter tool is used to add final shelters as facilities, and the Add Current Location tool is 
used to add the current location. Next, the Solve tool calculates the entire shortest route from a current location to each shelter. The final operation is the Select Data tool, which is used to store the routes as output to be displayed in a map server.

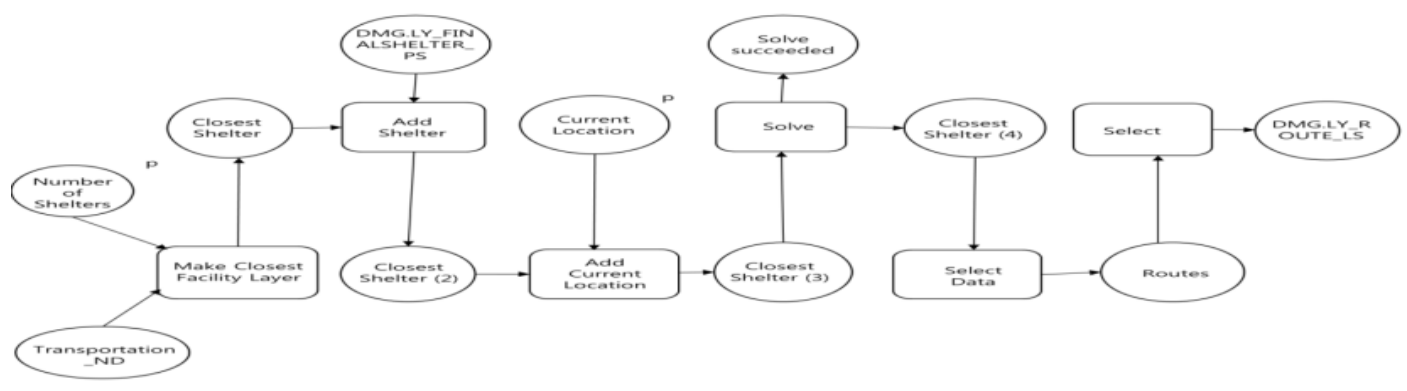

Figure 3. Diagram of the Evacuation Path Model

3.2.4. Case Study for Simulation of the Model: Bussan, the second capital city of South Korea, is surrounded by rivers, beaches, or mountains, where intensive rains fall down during summer seasons. We assume that civilians within the danger zones caused by sudden flooding and serious waves require some shelters. To provide the defined numbers of shelters, we calculate the shelter scores with the models suggested above.

Shelters should be located close to evacuation roads and in areas of suitable proximity to health facilities to provide medical aid during an evacuation period. Both proximity and shelter coverage are reclassified into six intervals based on distance and area using natural breaks. The natural break classes are based on natural groupings that best group similar values and that maximize the difference in classes. This classification is based on the Jenks' Natural Breaks algorithm [19].

The class interval depends on a defined danger zone. Shelters on Locations closer to major roads and hospitals are assigned to higher ratings as shown in Table 3 . In the case of shelter coverage of a danger zone, the wider the coverage is, the more suitable the shelter is. A thiessen proximal polygon is used to describe the area of influence of a point and to determine which shelter has the most coverage over a dangerous area.

Table 3. Factor Ratings Assigned to Each Interval

\begin{tabular}{|c|c|c|c|}
\hline Factor & Class Interval & $\begin{array}{l}\text { Number of } \\
\text { Existing Shelters }\end{array}$ & $\begin{array}{l}\text { Factor } \\
\text { Rating }\end{array}$ \\
\hline \multirow{6}{*}{ Proximity to health care facility } & $<136$ meters & 12 & 10 \\
\hline & $136-203$ meters & 13 & 8 \\
\hline & $203-294$ meters & 20 & 6 \\
\hline & $294-361$ meters & 16 & 4 \\
\hline & $361-431$ meters & 16 & 2 \\
\hline & $>=431$ meters & 17 & 0 \\
\hline \multirow{6}{*}{ Proximity to major road } & $<29$ meters & 48 & 10 \\
\hline & $29-56$ meters & 30 & 8 \\
\hline & $56-95$ meters & 15 & 6 \\
\hline & $95-160$ meters & 24 & 4 \\
\hline & $160-218$ meters & 23 & 2 \\
\hline & $>=218$ meters & 15 & 0 \\
\hline \multirow{6}{*}{$\begin{array}{l}\text { Coverage of each shelter in danger } \\
\text { zone }\end{array}$} & $<1,474 \mathrm{~m}^{2}$ & 1 & 0 \\
\hline & $1,474-6,496 \mathrm{~m}^{2}$ & 1 & 2 \\
\hline & $6,496-11,396 \mathrm{~m}^{2}$ & 1 & 4 \\
\hline & $11,396-23,751 \mathrm{~m}^{2}$ & 1 & 6 \\
\hline & $23,751-33,086 \mathrm{~m}^{2}$ & $\frac{1}{2}$ & 8 \\
\hline & $>=33,086 \mathrm{~m}^{2}$ & 2 & 10 \\
\hline
\end{tabular}

We investigate which shelters will be selected according to different weights with the assumption that we select four candidate shelters out of the shelter coverage 
points generated by the Thiessen polygon technique within a danger zone. In Figure 4, the letters indicate HF for the proximity to a health care facility, RF for proximity to a major road facility, and CF for proximity to shelter coverage facility. Some shelters within the coverage generated by the Thiessen tool are differently selected for each weight. The second shelter (SHT2 with $\mathrm{HF}=50, \mathrm{RF}=50, \mathrm{CF}=0$ ) is ranked the lowest because of the lowest proximity to health care facilities even though it has a coverage weight of zero for CF.

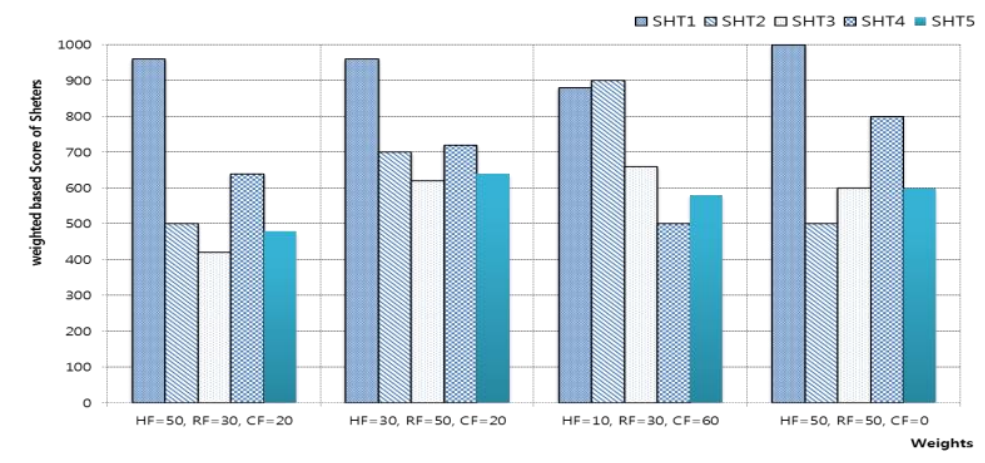

Figure 4. Selected Shelters According to Different Weights

\section{Implementation}

\subsection{Physical Architecture}

To develop Web and mobile applications to generate evacuation shelters and paths based OWS architecture, we design our system which consists of a spatial database server, a Web client, and Web, GIS and map servers as shown in Figure 5.

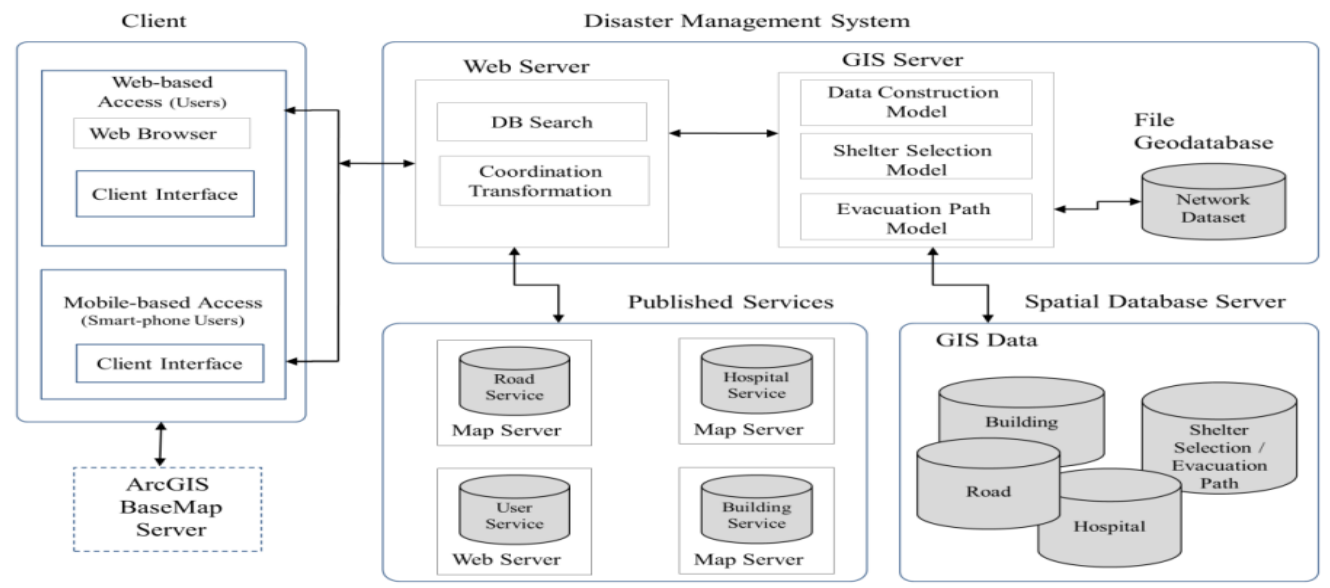

Figure 5. Physical Architecture for Evacuation Shelters and Paths

The Web clients work to display and query spatial data stored at remote locations that are only accessible by Internet or Intranet. The Smartphone Application is used by the individual to view the map and the shortest route from their location to a safe shelter. The Web Server processes requests from the Web client, by linking published Map Servers and the GIS server connected to the Spatial Database Server and Network Dataset to remotely process and analyse the spatial data model. The Published Services includes the shelter layer information with display field, feature type, extent renderer, and fields. In particular, the User Service is posted by 
managers or users of disaster-related organization such as police stations, governments, hospitals, or emergency centers. The GIS Server includes our three spatial models for selecting shelters and generating evacuation paths for each shelter.

\subsection{User Interface}

We developed a Web-based system to provide shelters and routes for evacuation. The GIS clients are developed using the Microsoft Silverlight as a platform for developing the Web application on an IIS and ArcGIS server domain. A mobile application is developed on the Android OS using map services.

The application has five panels for user interaction, including layer list, editor damage area, action panel, set current location, and detail panel as shown in Figure 6 : (1) The layer list panel shows the list of layers on the map and includes the functionality to set the opacity and visibility of the layers; (2) The Editor damage area panel enables users to draw and edit disaster damaged areas represented by a type of polygon on the map; (3) The action panel consists of two buttons, one for adding layers to the map using WMS and the other for initiating the shelter selection model. Users must select the number of shelters and a damaged area before pressing the "get shelter" button to generate the appropriate shelters.

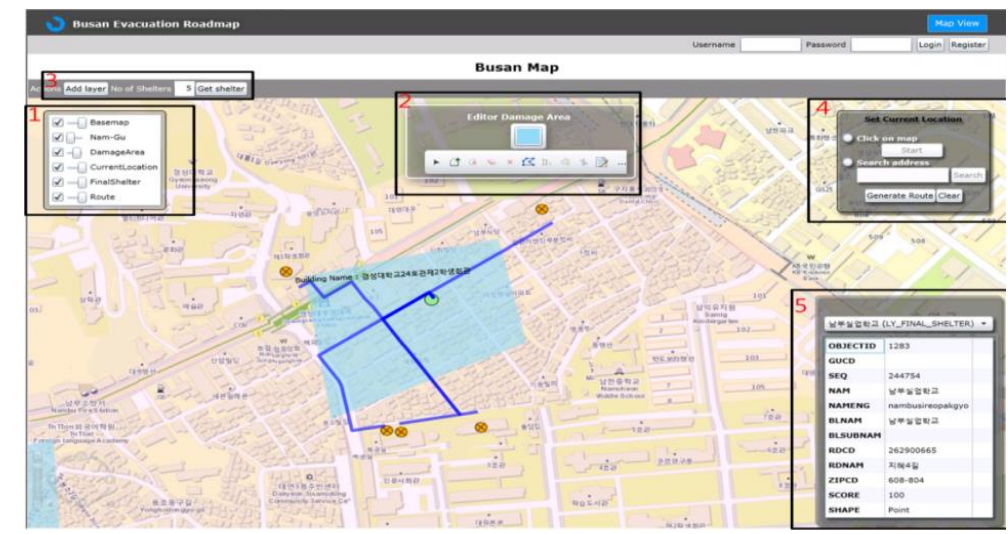

Figure 6. User Interface

The next two steps provide routes from the users' current location to each shelter selected: (4) Set Current Location enables users to set their own location for the evacuation path generation and to select the location by clicking on the map or by searching the location address using the building name. Generating Routes displays an evacuation path to each shelter on the Maps; (5) the detail panel consists of detailed information about a shelter such as building name, address, zipcode, and other pertinent information. Because the results are transferred using the REST Web Service in XML form, the evacuation path can also be viewed on a smartphone without having the full functionalities of the Web-based application.

\section{Conclusions}

In this paper, we propose a Web-based application for shelter and evacuation path selection. We provide the results of the proposed spatial model on the Web application using the OGC interface including Web Map Services. The model includes data construction, shelter selection, and an evacuation path model. The natural breaks method and a Thiessen tool are used to identity final shelters out of candidate shelters. This system provides different shelters for each danger zone based on the weights of three variables: proximity to health care facilities and major 
roads, and the coverage of each shelter in the danger zone. The shortest route is generated using the network analysis of ArcGIS.

The limitations of the study include: (1) not considering the attribution characteristics of the shelters and (2) the need for adaptive models for global disaster situations and zones defined by Thiessen polygon technique.

\section{Acknowledgements}

This work was supported by the Pukyong National University Research Abroad Fund in 2013(C-D-2013-0909).

\section{References}

[1] B. Karr and M. E. Hodgson, "A GIS-based model to determine site suitability of emergency evacuation shelter", Transaction in GIS vol. 12, no. 2 (2008), pp. 227-248.

[2] S. Shekhar, K. S. Yang, M.V. Venkata, L. Manikonda, D. Oliver, X. Zhou, B. George, S. Kim, J. M. R. Wolff and Q. Lu, "Experiences with evacuation route planning algorithms", International Journal of Geographical Information Science, vol. 26, no. 12, (2012), pp. 2253-2265.

[3] D. L. Niko, H. S. Hwang, Y. G. Lee and C. S. Kim, "Integrating user-generated content and spatial data into web GIS for disaster history", Studies in Computational Intelligence, vol. 365, (2011), pp. 245-255.

[4] L. Zhenlong, P. Y. Chaowei, W. Huayi, L. Wenwen and M. Lizhi, "An optimized framework for seamlessly integrating OGC web services to support geospatial sciences", International Journal of Geographical Information Science, vol. 25, no. 4, (2010), pp. 595-613.

[5] A. Mansourian, A. Rajabifard, M. J. Valadan Zoej and I. Williamson, "Using SDI and web-based system to facilitate disaster management", Computer \& Geosciences, vol. 32, (2006), pp. 303-315.

[6] V. Hristidis, S. C. Chen, T. Li, S. Luis and Y. Deng, "Survey of data management and analysis in disaster situations", The Journal of Systems and Software, vol. 83, (2010), pp. 1701-1714.

[7] M. Molina and S. Bayarri, "A multinational SDI-based system to facilitate disaster risk management in the andean community", Computers \& Geosciences, vol. 37, no. 9, (2011), pp. 1501-1510.

[8] A. Friis-Christensen, L. Roberto, L. Michael and O. Nicole, "Service chaining architectures for applications Implementing Distributed Geographic Information Processing", International Journal of Geographical Information Science, vol. 23, no. 5, (2009), pp. 561-580.

[9] C. Garnell, L. Diaz and M. Gould, "Service-oriented applications for environmental models reusable geospatial services", Environmental Modelling \& Software, vol. 25, no. 2, (2010), pp. 182-198.

[10] R. Vargas, M. Molina and S. Bayarri, A. Campos, "The Andean information system for disaster prevention and relief: SIAPAD an initiative for the development of a thematic SDI", Global Spatial Data Infrastructure conference, GSDI, vol. 10, (2008), pp. 25-29.

[11] G. Giuliani and P. Peduzzi, "The PREVIEW global risk data platform: a geoportal to serve and share global data on risk to natural hazards", Natural Hazards and Earth System Sciences, vol. 11, (2011), pp. 53-66.

[12] Y. L. Lee, H. Ishii and C. Tai, "Earthquake shelter location evaluation considering road structure", Proceedings on International Conference on Intelligent Systems Design and Applications, (2008), pp. 495-497.

[13] J. Sanyal and X. X. Lu, "Ideal location for lood shelter: a geographic information system approach", Journal of Flood Risk Management, vol. 2, no. 4, (2009), pp. 262-271.

[14] Z. Chen, X. Chen, Q. Li and J. Chen, "The temporal hierarchy of shelters: a hierarchical location model for earthquake-shelter planning”, International Journal of Geographical Information Science, vol. 27, no. 8, (2013), pp. 1612-1630

[15] D. Papinski and D. M. Scott, "A GIS-based toolkit for route choice analysis", Journal of Transport Geography, vol. 19, no. 3, (2011), pp. 434-442.

[16] E. Mohammadi, W. deVries and A. Mansourian, "Integration of multi-source spatial datasets via web services semi-Automatically and ontology based", World Applied Sciences Journal, vol. 3, (2008), pp. $127-135$.

[17] X. Meng, Y. Xie and F. Bian, "Distributed geospatial analysis through web processing service: a case study of earthquake disaster assessment", Journal of Software, vol. 5, no. 6, (2010), pp. 671-678.

[18] S. Zongyao and X. Yichun, "Design of service-oriented architecture for spatial data integration and its application in building web-based GIS systems", Geospatial Information Science, vol. 13, no. 1, (2010), pp. 8-15.

[19] G. Jenks, "The data model concept in statistical mapping", International Yearbook of Cartography (1967), pp. 186-190. 


\section{Authors}

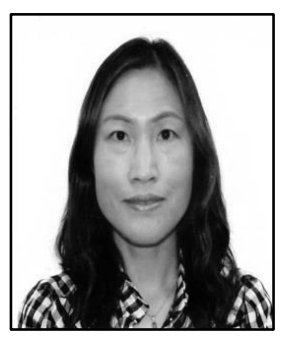

Hyun-Suk Hwang, She received the MS degree in Computer Science and the Ph.D. degree in Management Information System from Pukyong National University in Korea in 1995 and 2001 respectively. She worked the program of Post- Doc. at Kyungname University from 2001 to 2002, at University of Missouri, Kansas City (UMKC) from 2003 to 2004, and at Tongmyong University from 2006 to 2007. Her current research interests are in the area of LBS/GIS systems, ontology, data mining, wireless sensor networks, and urban disaster prevention systems.

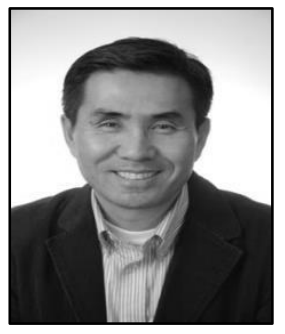

Il-Kyeun Ra, He holds a Ph.D. degree in Computer and Information Science from Syracuse University in 2001, M.S. degree in Computer Science from University of Colorado Boulder, and B.S. degree and M.S. degree in Computer Science from Sogang University. He was a Research Staff Member at the LG Information and Communications Research Center. He joined the department of Computer Science and Engineering at the University of Colorado Denver 2001. His main research interests include computer networks, developing adaptive distributed system software and high speed communication system software to support High Performance Distributed Computing Applications.

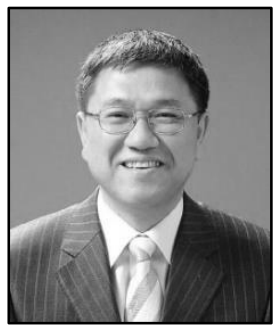

Chang-Soo Kim, He received a BS degree in Computer Science from Ulsan University, Korea, in 1979, and a MS degree in Computer Engineering and Ph.D. degree in Computer Engineering from Chungang University, Korea, in 1984 and 1991 respectively. He has been a professor at the department of IT Convergence and Application Engineering, Pukyong National University, Korea since 1992. His research interests are Operating Systems, Geographic Information System/Location Based Services, USN/RFID, and urban disaster prevention systems. 